\title{
Novel transcriptional targets of ETV6, a transcription factor frequently altered in childhood pre-B acute lymphoblastic leukemia
}

Camille Malouf $^{1,2}$, Karine Lagacé $^{1,2}$, Claire Drullion ${ }^{1}$, Sylvie Langlois ${ }^{1}$, Josiane Larose ${ }^{1}$, Ekat A. Kritikou ${ }^{1}$, Christia Sirard $^{1}$, Simon Drouin ${ }^{1}$ and Daniel Sinnett ${ }^{* 1-3}$

${ }^{1}$ Division of Hematology-Oncology, Sainte-Justine University Health Center Research Center, Montreal, Canada H3T 1C5 ${ }^{2}$ Department of Biochemistry

${ }^{3}$ Department of Pediatrics, Faculty of Medicine, University of Montreal, Montreal, Canada, H3T 1C5

Received: 23 September, 2016; Accepted: 10 October, 2016; Published: 20 October, 2016

*Corresponding author: Daniel Sinnett, Sainte-Justine University Health Center Research Center, 3175 chemin de la Côte-Ste-Catherine, Montreal (Quebec) Canada, H3T 1C5, Tel:+1-514-345-4931 ext.2990; E-mail: daniel.sinnett@umontreal.ca

\begin{abstract}
Pre-B Acute Lymphoblastic Leukemia (ALL), the most common hematological malignancy in children, represents $\sim 25 \%$ of all pediatric cancer cases. The most frequent genetic alteration associated with pre-B ALL is the $\mathrm{t}(12 ; 21)$ translocation, which results in the expression of the ETV6-RUNX1 chimera. The frequent deletion of the residual ETV6 allele leads to the complete loss of wildtype ETV6, a ubiquitously expressed Ets family transcriptional repressor with few known target genes. We had previously identified candidate ETV6 transcriptional targets using microarray gene expression profiling. Here we show, using chromatin immunoprecipitation experiments and reporter gene assays, that the sphingosine kinase 1 (SPHK1) and prostaglandin E2 receptor EP4 subtype (PTGER4) genes are direct ETV6 transcriptional targets. Furthermore, ETV6-mediated transcriptional repression of both genes requiresboth ETV6's pointed (PNT) and Erythroblast Transformation Specific (ETS) functional domains, and depends on Ets-Binding Sites (EBS) in the proximal promoter region of the target genes. Functional studies in leukemic cells implicated SPHK1 and PTGER4 in cell survival, proliferation, clonogenic capacity and migration. This study is one of the first to elucidate the functional role of ETV6 transcriptional targets and to suggest their role in childhood leukemogenesis.
\end{abstract}

\section{Introduction}

Acute Lymphoblastic Leukemia (ALL) is the most frequent pediatric cancer in children and accounts for $\sim 25 \%$ of all pediatric cancers [1]. Precursor B cell ALL (pre-B ALL), the predominant form of childhood ALL, has been associated with many genetic abnormalities including chromosomal translocations [2]. The $t(12 ; 21)$ translocation is the most common genetic aberration in childhood pre-B ALL, occurring in $25 \%$ of pre-B ALL cases [2]. This translocation leads to the formation of the ETV6RUNX1 chimera, an in-frame fusion of an ETV6 (erythroblast transformation-specific variant 6) allele with an allele of RUNX1 (runt-related transcription factor 1) [3, 4]. The expression of the ETV6-RUNX1chimera is under the control of the ETV6 promoter, which leads to the expression of a chimeric transcription factor composed of the N-terminal part of ETV6 fused to the near complete RUNX1 protein.

ETV6 is a ubiquitously expressed transcription factor of the Ets family [5]. Unlike most Ets transcription factors that function as transcriptional activators, ETV6 has been shown to act as a transcriptional repressor [6]. ETV6 is essential for embryonic development as ETV6 knockout mice succumb to severe defects in the vascular network of the yolk sac and to increased apoptosis of mesenchymal and neural tissues [7]. Using inducible gene disruption and ETV6\% chimeric mice, ETV6 was shown to be involved in the survival/homing of the hematopoietic stem cells and in the differentiation of the megakaryocytic precursors within the bone marrow microenvironment $[8,9]$.

ETV6 has a C-terminal ETS DNA-binding domain and a pointed (PNT) helix-loop-helix domain required for proteinprotein interactions [6]. The ETS domain has been shown to recognize a consensus Ets-binding site (EBS), which consists of a core GGAA/T sequence with adjacent purine-rich sequences [10]. The PNT domain is involved in the interactions of Ets transcription factors with other proteins [10]. Unlike other Ets proteins, ETV6 has been shown to homodimerize in vivo. This interaction requiresits PNT domain, which is also required for its transcriptional repression activity [6]. The central domain of ETV6 is also implicated in protein-protein interactions with members of the SMRT/N-CoR/mSin3A/HDAC corepressor complexes [11-13].

Several lines of evidence indicate that the inactivation of ETV6 is one of the early events in leukemogenesis of ALL [14]. Studies in transgenic mice [15, 16], zebrafish [17], and humanized NOD/ SCID $\gamma$ mice transplanted with cord blood [18] have clearly shown that the ETV6-RUNX1 translocation is not sufficient for pre-B ALL development, indicating that additional steps are required for leukemic transformation [16]. The non-translocated ETV6 allele is inactivated through deletion (loss of heterozygosity (LOH)) in up to $90 \%$ of pre-B ALL cases carrying $\mathrm{t}(12 ; 21)$ [19, 20]. Interestingly, ETV6 expression is also absent in patients 
that do not have $\mathrm{LOH}$ in the residual allele, suggesting that other mechanisms contribute to the ETV6 inactivation [21, 22]. Consistent with its tumor suppressor activity, overexpression of ETV6 in Ras-transformed fibroblasts inhibits cell growth and enhances apoptosis in vitro and suppresses tumor formation in nude mice [23-25]. Thus, the complete inactivation of ETV6 appears to be required for leukemogenesis [26].

A complete understanding of the impact of the loss of ETV6 in the initiation and progression of leukemia requires the identification of its downstream target genes. Very few transcriptional targets of ETV6 are currently known: MCSFR (macrophage colony-stimulating factor receptor) [27], GpIb $\alpha$ and GPIX (platelet glycoprotein Ib alpha chain and IX) [28], stromelysin-1/MMP-3 (matrix metalloprotein-3) [23] and the anti-apoptotic protein $B C L-X_{L}[25]$. Using a microarray-based approach, we had previously identified several putative ETV6regulated genes, including SPHK1 (sphingosine kinase 1) and PTGER4 (prostaglandin E2 receptor EP4 subtype) [29].

In the present study, we investigated ETV6-mediated transcriptional regulation on the proximal promoters of SPHK1 and PTGER4 using Chromatin Immunoprecipitation (ChIP) and gene reporter assays. We show that SSPHK1 and PTGER4 are direct ETV6 transcriptional targets with ETV6-mediated transcriptional regulation requiring consensus EBS in the proximal promoter region. Furthermore, functional studies revealed that deregulated expression of these novel ETV6 transcriptional targets in Reh, an ETV6-deficient $\mathrm{t}(12 ; 21)$ pre-B leukemia cell line, play a role in the pathophysiology of childhood pre-B ALL.

\section{Materials and Methods \\ Cell culture}

HeLa cells (immortalized cervical cells from an adenocarcinoma patient, ATCC \#CCL-2 ${ }^{\mathrm{TM}}$ ) were cultured in DMEM 10\% FBS
(Wisent) at $37^{\circ} \mathrm{C}, 5 \% \mathrm{CO}_{2}$. Jurkat cells (immortalized leukemic $\mathrm{T}$ lymphocytes from a 14 years old patient, kindly provided by Dr. José Menezes), IM-9 cells (Epstein-Barr virus-transformed B lymphoblast from a multiple myeloma patient, ATCC \#CCL-159) and Reh cells (immortalized $\mathrm{t}(12 ; 21)^{+}$leukemic pre-B lymphocytes, ATCC \#CRL-8286 ${ }^{\mathrm{TM}}$ ) were cultured in RPMI-1640 10\% FBS (Wisent) at $37^{\circ} \mathrm{C}, 5 \% \mathrm{CO}_{2}$. Unless otherwise specified, cell culture conditions were the same for all experiments.

\section{Constructs}

SPHK1 and PTGER4 promoters were amplified by PCR (Platinum Taq, Invitrogen) and subcloned in the pGL3-basic vector (Promega) upstream of the Firefly luciferase gene. The proximal promoter region tested were as follows: SPHK1 promoter -858/+115 and PTGER4 promoter -855/+601 [Figure 2A]. Full-length ETV6 was subcloned downstream of the pcDNA3.1 vector's (Invitrogen) CMV promoter. Mutation of consensus EBS (GGAA $\rightarrow$ CCAA) was done using the Quick Change Multi-Site Directed Mutagenesis kit (Stratagene). The PNT and ETS domains of ETV6 were removed by restriction endonuclease after inserting, respectively, a PacI or KpnI restriction site at each end of the domain of ETV6 subcloned in pcDNA3.1. The pLKO.1 constructs harboring the SPHK1 shRNA and PTGER4 shRNA were purchased from Open Biosystems (RHS4533, RHS4533 and EHS1001-43247). The integrity of all constructs was verified by direct sequencing of both strands with the ABI 3730 automatic sequencer. Primers, shRNA and scrambled shRNA sequences are presented in Table 1.

\section{Gene reporter assays}

HeLa and Jurkat cells were transfected using lipofectamine $\mathrm{e}^{\mathrm{TM}}$ 2000 (Invitrogen) with a combination of constructs: promoter constructs in pGL3-basic vector (Promega), pRL-CMV vector

Table 1: Primers used for molecular cloning, site-directed mutagenesis and chromatin immunoprecipitation

\begin{tabular}{|c|c|}
\hline Name & Sequence (5' to 3 ') \\
\hline \multicolumn{2}{|l|}{ Cloning : promoters } \\
\hline proSPHK1-858.R & agaggtcgacccggattcctggagcaag \\
\hline proSPHK1-617.F & agaggtcgaccgcgtccctactggcctc \\
\hline proSPHK1-351.GW.R & ggggaccactttgtacaagaaagctgggtacacgagttcgcgcgga \\
\hline proSPHK1-168.GW.R & ggggaccactttgtacaagaaagctgggtttgccgcttcctaggaccc \\
\hline proSPHK1+115.F & agagaagcttcccacgtctgagggactg \\
\hline proSPHK1+115.GW.F & ggggacaagtttgtacaaaaaagcaggctcccacgtctgagggactg \\
\hline proIL18-1807.F & agagacgcgtcccaagggtctgggaaatac \\
\hline proIL18-1110.F & agagacgcgtttcccgaaatgaaaacccta \\
\hline proIL18-667.F & agagacgcgttccetctccccaagcttact \\
\hline proIL18-311.F & agagacgcgtgtgctgaagtgtgaccagga \\
\hline proIL18-174.GW.F & ggggacaagtttgtacaaaaaagcaggctagagccccaacttttacggaag \\
\hline proIL18+185.R & agagacatctaagcgatctggaaggtctga \\
\hline proIL18+79.GW.R & ggggaccactttgtacaagaaagctgggttgcactgggagacaattccttg \\
\hline proPTGER4-855.F & agagacgcgtaagctcacgctaccctttca \\
\hline proPTGER4-337.GW.F & ggggacaagtttgtacaaaaaagcaggctaaacggtcgccggtttcagt \\
\hline proPTGER4+601.R & agagagatctctgcgttctctacccgtta \\
\hline proPTGER4+387.R & agagagatctccgaggtcagagttgcc \\
\hline proPTGER4+178.R & agagagatctagctctggctgccctc \\
\hline
\end{tabular}




\begin{tabular}{|c|c|}
\hline proPTGER4+178.GW.R & ggggaccactttgtacaagaaagctgggtagctctggctgccctcc \\
\hline proLUM-829.F & agagacgcgtccacaagtctcactgctaaaatc \\
\hline proLUM-583.F & agagacgcgtgcctttgggagattattac \\
\hline proLUM-411.F & agagacgcgtgaattcctaaacgaaatcc \\
\hline proLUM+332.R & agagctcgaggcagatgcactatggacaaga \\
\hline \multicolumn{2}{|l|}{ Cloning : Site-directed mutagenesis } \\
\hline proSPHK1.mEBS-158 & ttgccgcttggtaggacccgggcgggaacc \\
\hline proSPHK1.mEBS-104 & ggccgtgccccaggcggcgcgctgagg \\
\hline proSPHK1.mEBS-84 & gctgaggccccaggcgggcagaggccg \\
\hline proSPHK1.mEBS+60 & gtttgaggccccagggagcgaggccggg \\
\hline proIL18.mEBS-157 & ggcacagagccccaacttttacccaagaaaagatttc \\
\hline proIL18.mEBS-79 & gccactttatttcgacttggattgccctaggaaagagcc \\
\hline proIL18.mEBS-3 & ccctccaccttcttggtcattctctccccag \\
\hline proIL18.mEBS+61 & ctgcctggacagtcagcaaccaattgtctcccagtgc \\
\hline proPTGER4.mEBS-305 & cagtggtggccccactccaagccgagttcagg \\
\hline proPTGER4.mEBS-97/-92 & gctccgcccccttggaatggggccaatgggc \\
\hline proPTGER4.mEBS-55/-49 & cgcggtttgcctgggcctgggccagggaaacttgg \\
\hline proPTGER4.mEBS+35 & cagcccgagaccaagatgaacagccccaggc \\
\hline proLUM.mEBS-152 & gggcaaagggattcaattggtctgagtctgttcatttgcg \\
\hline proLUM.mEBS-126 & gggattcaattcctctgagtctgttcatttgcgttttggtgaaattatttgccc \\
\hline proLUM.mEBS+13 & aatgttctcacagtgagcttggttatttgaagcaggactc \\
\hline proLUM.mEBS+277 & ggcagccagttgggtcctgacagagttcacagc \\
\hline ETV6.APNT.PacI & cacctgcgcttgcagttaattaactggagcagggatgac \\
\hline ETV6.APNT.PacI & gtgctctatgaactccttcagcatttaattaagcagaggaaacctcgg \\
\hline 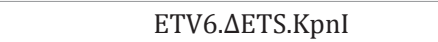 & caggccatgcccattggtaccatagcagactgtagactg \\
\hline 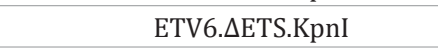 & gtttatgaaaaccccagatggtaccatgagtggccgaacagac \\
\hline \multicolumn{2}{|c|}{ Cloning : shRNA and scrambled shRNA } \\
\hline $\begin{array}{c}\text { SPHK1 shRNA (shSPHK1) } \\
\text { (Clone ID: TRCN0000036964) }\end{array}$ & ccgggcaggcatatggagtatgaatctcgagattcatactccatatgcctgctttttg \\
\hline $\begin{array}{l}\text { scrambled SPHK1 shRNA } \\
\text { (scSPHK1) }\end{array}$ & ccgggagtccctacgcctcatacatctcgagatgtatgaggcgtagggactcttttt \\
\hline $\begin{array}{l}\text { PTGER4 shRNA (shPTGER4) } \\
\text { (Clone ID: TRCN0000000204) }\end{array}$ & ccgggtactgtttctggacccttatctcgagataagggtccagaaacagtacttttt \\
\hline $\begin{array}{l}\text { scrambled PTGER4 shRNA } \\
\text { (scPTGER4) }\end{array}$ & ccgggctctcttcctatgctagactctcgagagtctagcataggaagagagcttttt \\
\hline \multicolumn{2}{|l|}{ Chromatin Immunoprecipitation } \\
\hline 14834 ChIP.F (+) & ttgcccaatcatttcctttc \\
\hline 14834 ChIP.R (+) & cctccatgttgactgacacg \\
\hline Negative ChIP.F (-) & atggttgccactggggatct \\
\hline Negative ChIP.R (-) & tgccaaagcctaggggaaga \\
\hline proSPHK1-849. ChIP.F & actggcctccaaagaagtga \\
\hline proSPHK1-679. ChIP.R & ggagaggaggcttgacagtg \\
\hline proIL18-82. ChIP.F & ttccattgccctaggaaaga \\
\hline proIL18+78. ChIP.R & gcactgggagacaattcctt \\
\hline proIL18-1110.ChIP.F & ttcccgaaatgaaaacccta \\
\hline proIL18-958.ChIP.R & agcaaggtggcattttgttt \\
\hline proPTGER4-103.ChIP.F & gcttagccgctcctgaact \\
\hline proPTGER4+76.ChIP.R & agcgcagacaccgtatttct \\
\hline proPTGER4-258.ChIP.F & gcttagccgctcctgaact \\
\hline proPTGER4-485.ChIP.R & agcgcagacaccgtatttct \\
\hline proLUM-44.ChIP.F & gccacagccacagatgtaaa \\
\hline proLUM+144.ChIP.R & taacgaagtgcaggtgaacg \\
\hline proLUM-638.ChIP.F & ctcatttgctgaaaccatgc \\
\hline proLUM-418.ChIP.R & aaccttacttggggagagca \\
\hline
\end{tabular}


(Promega) to normalize the transfection efficiency and ETV6 constructs in pcDNA3.1 vector. $1 \mu \mathrm{g} / \mathrm{mL}$ leucoagglutinin and $50 \mathrm{ng} / \mathrm{mL}$ phorbol 12-myristate 13-acetate were added to each reaction 4-hour after transfection (Sigma Aldrich) in Jurkat cells. Luciferase activity was measured 48-hour later using the DualLuciferase ${ }^{\circledR}$ Reporter Assay System (Promega) and LLMAX ${ }^{384}$ LUMinometer (Molecular Devices).

\section{Chromatin immunoprecipitation assays}

HeLa cells with inducible ETV6-HA [29] were incubated for 48-hour with $2 \mu \mathrm{g} / \mathrm{ml}$ doxycycline and IM9 cells expressing a lentivirus harboring ETV6-HA (pLenti-ETV6-HA) were cross-linked with $1 \%$ formaldehyde for 10 minutes at room temperature, glycine stopped, lysed, and sonicated to obtain 200$1500 \mathrm{bp}$ DNA fragments. After immunoprecipitation with an antiHAantibody (Santa Cruz Biotechnology sc-7392), the precipitates were reverse cross-linked and treated with proteinase $\mathrm{K}$. The purified endogenous DNA was analysed by quantitative real-time PCR. Experimental controls were selected according to a previous ChIP-chip experiment conducted by our group (unpublished data): Negative maps to an exon-intron junction region of the chromosome-associated protein D2 (NCAPD2), 14834 maps to the proximal promoter of the activator of basal transcription 1 (ABT1) gene and 10505 maps to the proximal promoter of the cAMP responsive element binding protein 1 (CREB1).

\section{Lentiviral transduction}

$1 \times 10^{6}$ Reh cells were infected at a multiplicity of infection of 1 in RPMI-1640 10\% FBS $8 \mu \mathrm{g} / \mathrm{mL}$ polybrene (Sigma-Aldrich). Forty-eight hours later $1 \mu \mathrm{g} / \mu \mathrm{L}$ puromycin (Sigma-Aldrich) was added to enrich for transduced cells.

\section{Quantitative PCR and immunoblot}

Quantitative real-time PCR of mRNA was done as previously described [29]. For Western blots, $15 \times 10^{6}$ Reh cells were homogenized in RIPA buffer and quantified using a Lowry assay (BioRad). Proteins were denatured in sample buffer with SDS (2\%) and 2-mercaptoethanol (2 mM), separated on a $12 \%$ SDSPAGE and electroblotted to a nitrocellulose membrane (Roche). Non-specific binding sites were blocked using skimmed milk followed by hybridization with the primary antibodies (SPHK1: Abcam ab-56414; PTGER4 and GAPDH: Santa Cruz Biotechnology sc-13602 and sc-31915, respectively) and the secondary antibodies (Santa Cruz Biotechnology sc-166574 or sc-48167). Enhanced chemiluminescence (Pierce) allowed protein detection and their subsequent quantification by densitometry (Adobe Photoshop 7.0.1).

\section{Cell proliferation}

Cell Proliferation Reagent WST-1 (Roche) and cell counting was used to study Reh cell proliferation. Reh cells were seeded at $0.4 \times 10^{5}$ cells $/ \mathrm{mL}$ and grown for four days without renewaing the culture medium. Formazan dye absorbance was measured at $450 \mathrm{~nm}$ with a reference at $750 \mathrm{~nm}$.

\section{DNA damage-induced apoptosis and cell cycle}

Reh cells were seeded at a density of $4 \times 10^{5}$ cells/mL in 24- well plates for 12 hours and treated with $2 \mu \mathrm{M}$ camptothecin for 4 hours (Tocris Bioscience). Harvested cells were stained using the FITC AnnexinV/Dead Cell apoptosis kit (Invitrogen). Cell cycle analysis was done using the FITC BrdU Flow Kit (BD Pharmigen) where $4 \times 10^{5}$ Reh cells were synchronized for 24hour by serum-starvation and cultured in RPMI 1640 10\% FBS medium for another 24-hour. Stained cells were captured and analyzed using BD Biosciences FACS Aria and BD FACSDiva software, respectively.

\section{Transmigration assays}

Migration of Reh cells was performed for 2-hour in a ChemoTx $^{\circledR} 3.2$ mm diameter 96 well-plate (NeuroProbe) with an input of $2 \times 10^{5}$ cells and CXCL12 as a chemoattractant (ProSpec). Cell quantification was done using the Cell Proliferation Reagent WST-1 (Roche) or by cell count using a Coulter counter (Beckman).

\section{Clonogenic assays}

After 2 weeks of selection with $1 \mu \mathrm{g} / \mu \mathrm{L}$ puromycin, 500 Reh cells infected with lentivirus were plated in quadruplicate in 1 $\mathrm{ml}$ of HSC-CFU Basic Human medium (Miltenyi Biotec inc) (1.2\% methylcellulose, 30\% FBS, 1\% BSA, 0.1mM 2-mercaptoethanol, and $2 \mathrm{mM}$ L-glutamine) onto $35 \mathrm{~mm}$ tissue culture dishes and incubated for 18 days in a humidified atmosphere at $37^{\circ} \mathrm{C}$ and $5 \% \mathrm{CO}_{2}$ [52]. Colonies consisting of $>40$ cells were counted using an inverted microscope.

\section{Statistical analysis}

Graphs and statistical analysis were done using the GraphPad 5.0 software. For all experiments, unless otherwise mentioned, statistical significance was indicated as follows: ${ }^{*}$ : $p \leq 0.05,{ }^{* *}$ : $p$ $\leq 0.01,{ }^{* * *}: \mathrm{p} \leq 0.001$.

\section{Results}

\section{ETV6 expression induces a tumor suppressor phenotype in lymphoblastic cells}

ETV6 has previously been shown to have tumor suppressor capabilities in NIH-3T3 cells [23-25]. To determine whether ETV6 has tumor suppressor activity in human lymphoblastic cells, we overexpressed ETV6 via lentivirus infection in REH cells, with high levels of ETV6 protein in infected cells confirmed by Western blot [Figure 1A, inset]. ETV6 overexpression reduced cell proliferation and clonogenic potential of Reh cells compared to mock infected cells [Figure 1A, B], suggesting that loss of ETV6 can increase the proliferative capacity of $\mathrm{t}(12 ; 21)$-positive pre-B leukemia cells.

\section{Etv6 mediates transcriptional repression of SPHK1 and ptger4 via its PNT and ETS functional domains}

We have previously identified several genes that were deregulated early after ETV6-induced expression using microarray expression profiling [29]. Two of these genes, SPHK1 and PTGER4, were retained for further analysis. To examine whether ETV6 can bind to these promoters endogenously, we first conducted ChIP assays in HeLa cells overexpressing HA- 
tagged ETV6 (ETV6-HA). Quantitative PCR (qPCR) analysis indicated that there was significant enrichment of chromatinbound ETV6 to genomic fragments encompassing the proximal promoter regions of SPHK1 and PTGER4 [Figure 2A] in HeLa cells when using an antibody against HA compared to a non-specific control antibody [Figure 2B]. Similar ChIP results were obtained using the IM-9 B lymphoblastoid cell line for the promoters of SPHK1 and PTGER4 [Figure 2C]. These results indicate that ETV6 can form a chromatin complex on the promoters of its target genes.

To examine the ETV6-dependent modulation of these genes, we used gene reporter assays in HeLa and Jurkat, two highly transfectable cells lines, that were co-transfected with a pcDNA3.1 expression vector overexpressing wildtype ETV6 or versions lacking either the PNT domain, involved in proteinprotein interaction, or the ETS domain, required for DNA binding at the consensus EBS [Figure 3A]. An artificial promoter containing three consensus EBS located upstream of the thymidine kinase minimal promoter $\left(\mathrm{EBS}_{3} \mathrm{tk}\right)$ was used as control for ETV6-mediated transcriptional repression. Following ETV6 overexpression, the activity of $\mathrm{EBS}_{3}$ tk was dramatically reduced in both HeLa and Jurkat cells [Figure 3B,C]. Previous studies on ETV6 transcriptional targets have shown that ETV6 requires the PNT and ETS functional domains to repress transcription [23, $25,27,28]$, which we confirmed in our system in both cell lines [Figure 3B, C].

To assess the ETV6-mediated transcriptional repression of the target genes, we subcloned a $\sim 2 \mathrm{~kb}$ region of the SPHK1 and PTGER4 proximal promoters along with a region downstream of the transcription start site that contains putative EBS into the pGL3 reporter vector [Figure 3A]. Overexpression of ETV6 in both cell lines resulted in significant transcriptional repression of all reporter constructs [Figure 3B, C], indicating that ETV6 can mediate transcriptional regulation of SPHK1 and PTGER4. Deletion of either PNT or ETS domain resulted in the loss of ETV6-mediated repression of these target genes in both cell lines [Figure 3B, C]. These findings indicate that both ETS and PNT domains, through direct binding to the promoter and protein-protein interactions, respectively, are required for ETV6mediated repressive function.

\section{ETV6 recognizes consensus EBS for transcriptional repression}

Similar to other Ets family members such as ETS1 and FLI1, ETV6 mediates transcriptional regulation by binding to a consensus EBS [30-32]. Close examination of the SPHK1 and PTGER4 proximal promoter regions using the Transfac bioinformatics tool [33] predicted many consensus EBS. To delineate the minimal region of the proximal promoter required for ETV6-mediated repression, truncated versions of the SPHK1 and PTGER4 promoters [Figure 2A] were subcloned in the pGL3 reporter vector. Co-transfection of the various reporter constructs with ETV6 in both HeLa and Jurkat cell lines allowed us to identify these regions:- $168 /+115$ for the SPHK1 promoter and $-337 /+178$ for the PTGER4 promoter (data not shown).
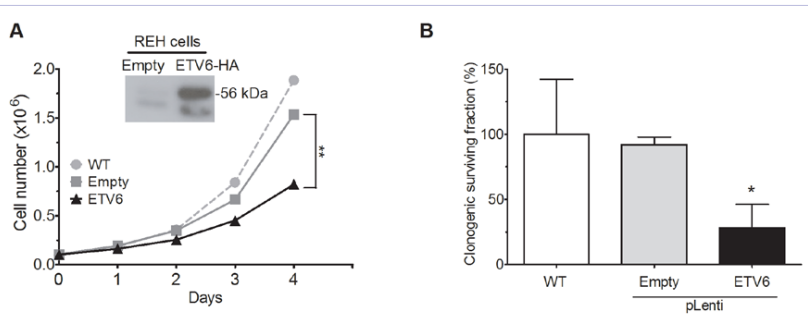

Figure 1: Tumor suppressor activity of ETV6 in Reh lymphoblastic cell line. (A) Overexpression of ETV6 in the Reh ETV6-deficient cell line inhibits cell growth. Cell proliferation assay of Reh cells (WT) stably infected with an empty lentivirus (Empty) or expressing ETV6-HA (ETV6) (inset). Cell counts were performed on triplicate samples and a representative growth curve from three independent experiments is shown. Statistical differences between the linear regression slopes ${ }^{* *}$ ) were assessed (days 0 to 4 ) using pLenti-empty as a reference. (B) Expression of ETV6 decreases the clonogenic potential of Reh cells. The clonogenic surviving fraction corresponds to the plating efficiency of the lentivirus-infected cells normalized to the plating efficiency of the uninfected (WT) Reh cells (expressed in percentage). Colony counts were performed in triplicate and shown is a representative clonogenic assay from three independent experiments. Statistical significance was assessed using one-way ANOVA with Bonferroni's multiple comparison post-test.

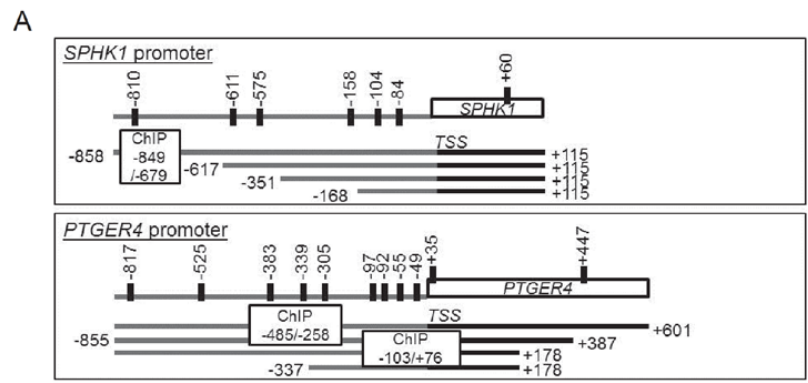

B

HeLa
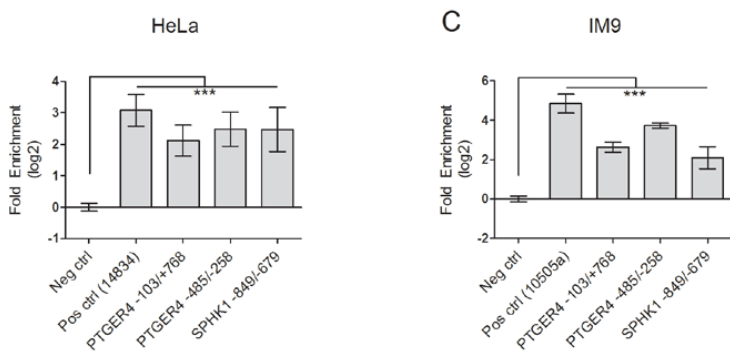

Figure 2: ETV6 binds SPHK1 and PTGER4 proximal promoters. (A) Schematic diagrams depicting the promoter regions of SPHK1 and PTGER4 used for chromatin immunoprecipitation (ChIP) and reporter gene assay experiments. The transcription start site (TSS) corresponds to position +1 . ETS binding site (EBS) predictions by Transfac are indicated by the thich black vertical lines. ChIP of the indicated promoter region in the HeLa (B) or the IM-9 lymphoblastoid (C) cell lines overexpressing HA-tagged ETV6. ETV6 binding enrichment is depicted as the fold enrichment of the HA antibody relative to the pre-immune serum immuno-precipitate, normalized to the negative control. Results were calculated using the $\Delta$ CT log values (mean $\pm \mathrm{SE}$ ). Statistical significance was assessed using one-way ANOVA with a Dunnett's multiple comparison post-test ( $\mathrm{n}=2$, in triplicates). Statistical significance: $\mathrm{p}<$ $0.05^{*}, \mathrm{p}<0.01^{* *}, \mathrm{p}<0.001^{* * *}$. 
Changing EBS consensus sequences from GGAA/T to CCAA/T is sufficient to abolish their function [34]. Using sitedirected mutagenesis, we tested the ability of the various EBS to mediate ETV6-induced transcriptional repression in the minimal promoters of each gene. The SPHK1 minimal promoter has four putative consensus EBS: $-158 /-104 /-84 /+60$. Mutation of any of these resulted in loss of ETV6-mediated repression in both HeLa and Jurkat cells [Figure 4A, B]. The minimal PTGER4 promoter has six predicted consensus EBS: -305/-97/-92/-55/-49/+35. Because of the proximity of EBSs $-97 /-92$ and of $-55 /-49$, these pairs were mutated simultaneously. All EBS mutant constructs, with the exception of -305, abolished ETV6-dependant transcriptional repression [Figure 4C, D]. These results indicate that ETV6 mediates transcriptional inhibition by binding to EBS in the proximal promoter region of both these genes.

\section{SPHK1 is a positive regulator of leukemic cell survival, proliferation, and migration}

To assess the biological impact of ETV6 transcriptional targets, we knocked down their expression in the ETV6deficient / t(12;21)-positive Reh cell line [35] and monitored cell proliferation, DNA damage-induced apoptosis, clonogenic potential, and cell migration. SPHK1 mRNA was reduced by $\sim 40 \%$ by infecting Reh cells with lentiviruses expressing a shRNA targeting its sequence (shSPHK1, Figure 5A). Western blot analysis confirmed this knockdown (data not shown). A scrambled sequence was used as a negative control (scSPHK1).

The proliferative properties of Reh cells were measured using the WST-1 colorimetric assay. SPHK1 knockdown resulted in a significantly slower proliferation of Reh cells, compared to control over a four day period (linear regression $y$ intercept $\mathrm{p}=0.032$ ) [Figure 5B]. However, downregulation of SPHK1 had no detectable impact on the cell-cycle distribution of a synchronized cell population 24 hours post-release (data not shown). These results are consistent with the well characterized role of the downstream effector of SPHK1, Sphingosine-1-Phosphate (S1P), on cell proliferation [36]. Indeed, higher levels of SPHK1 allow for more sphingosine to be converted into S1P via its lipid kinase activity, which results in enhanced cellular proliferation [37]. Sphingosine can also be transformed into pro-apoptotic ceramide via ceramide synthase [38]. Thus, SPHK1 levels are believed to act as a survival rheostat that balances the cellular level of the pro-apoptotic ceramide with the anti-apoptotic, pro-proliferation S1P signal. The role of SPHK1 on apoptotic pathways was therefore examined in Reh cells treated with camptothecin, a DNA damaging agent inhibiting the DNA repair ability of the DNA topoisomerase I. Downregulation of SPHK1 increased the sensitivity of Reh cells to camptothecin-induced apoptosis [Figure 5C].

The clonogenic potential of leukemic cell lines kept in suspension culture is reflected in the proportion of cells capable of maintaining in vitro cell growth. Indeed, the higher the clonogenic potential of a cell line, the higher the number of cells capable of autonomous cell growth. To examine the role of SPHK1 on the clonogenic potential of Reh cells colony formation in methylcellulose was assessed on cells expressing SPHK1 shRNA. Downregulation of SPHK1 considerably decreased the clonogenic potential of Reh cells compared to cells expressing scrambled control shRNA [Figure 5D].

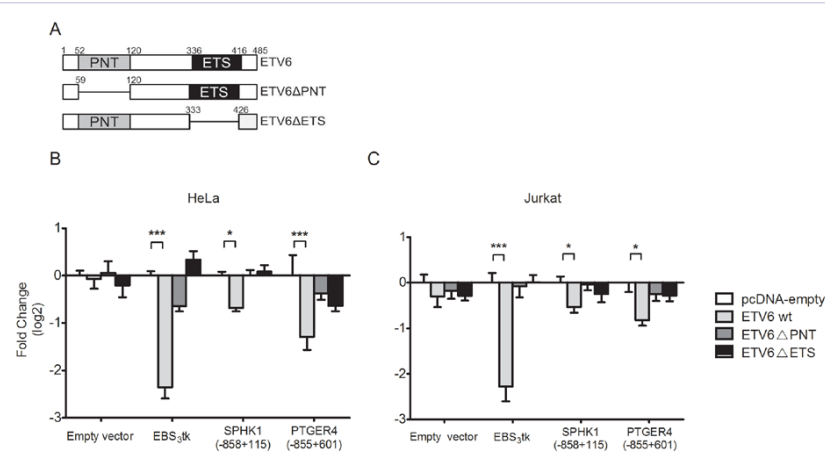

Figure 3: ETV6-dependent transcriptional repression requires both the ETS and PNT functional domains. (A) A schematic representation of the coding sequence of wild-type ETV6 and its deleted versions (ETV6 $\triangle$ PNT and ETV6 6 ETS). Amino acid positions are indicated. Reporter gene assays conducted in HeLa (B) and Jurkat (C) cell lines. "Empty vector" is the promoter-less pGL3-basic luciferase reporter vector and "EBS $\mathrm{tk}_{3}$ " contains 3 EBS and a thymidine kinase minimal promoter (ETV6 transcriptional repression positive control). The luciferase value is depicted as the firefly luciferase value of the pGL3-basic reporter gene vector relative to the Renilla luciferase value of the pRL-CMV control vector normalized to the cells transfected with empty pcDNA3.1 vector. Statistical significance was assessed using two-way ANOVA with Bonferroni's multiple comparison post-test ( $\mathrm{n}=3$, in triplicates).
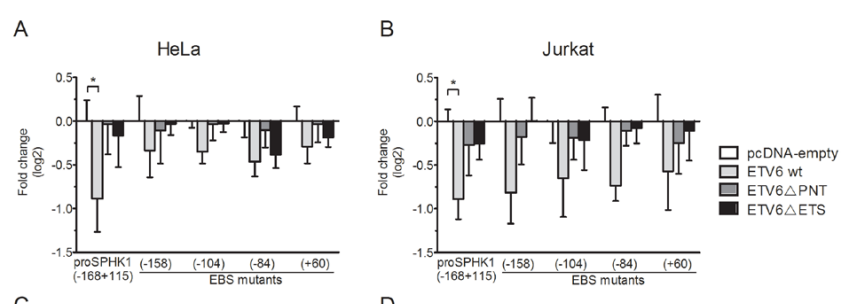

D
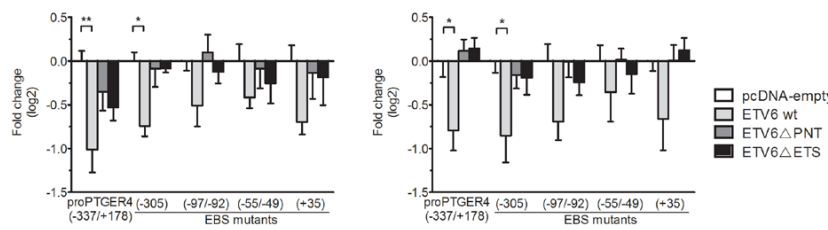

Figure 4: EBS consensus sites are involved in the ETV6-mediated transcriptional repression. Site-directed mutagenesis of predicted consensus EBS located in the basal promoters of SPHK1 (A, B) and PTGER4 (C, D) were performed on the corresponding luciferase reporter constructs. Gene reporter assays were conducted in HeLa and Jurkat cell lines. The luciferase value is depicted as the firefly luciferase value of the pGL3-basic reporter gene vector relative to the Renilla luciferase value of the pRL-CMV control vector normalized to the cells transfected with empty pcDNA3.1 vector. Statistical significance was assessed using two-way ANOVA with Bonferroni's multiple comparison post-test ( $\mathrm{n}=3$, in triplicates). 
Finally, we assessed the role of SPHK1 on the migration potential of leukemic cellsusing the CXCL12 chemoattractant in a transmigration assay. CXCL12 is the ligand for CXCR4, a G-protein coupled receptor expressed in pre-B leukemic cell lines [39]. SPHK1 downregulation reduced the migration potential of Reh cells at low CXCL12 concentration [Figure 5E], indicating that higher expression of SPHK1 can be favourable to the mobilisation of leukemic progenitors.

These results indicate SPHK1 is an important regulator of cell survival, proliferation, and migration in leukemic cells and suggest that ETV6, by repressing its expression, acts as a tumor repressor.

\section{PTGER4 expression promotes leukemogenesis}

PTGER4 is a G-protein coupled receptor responding to its ligand prostaglandin E2 (PGE2). To assess the functional role of PTGER4 during leukemogenesis its expression was knocked down by $\sim 65-70 \%$ using shRNA infection, as detailed above [Figure 6A]. Western blot analysis confirmed this knockdown (data not shown). PTGER4 downregulation, with both shRNAs, led to a significant decrease in clonogenic capacity and proliferation rate of Reh cells [Figure 6B], D but did not affect the cell cycle distribution of synchronized Reh cells over a 24-hour period (data not shown). Furthermore, PTGER4 repression inhibited CXCL12-induced migration Figure 6E. Interestingly, it had no effect on camptophecin-induced apoptosis [Figure 6C].

These data were confirmed by specifically inhibiting PTGER4 with the L-161,982 antagonist, which prevents PTGER4-mediated signaling [40]. Consistent dose-dependent growth inhibition was observed in the presence of PTGER4 inhibitor [Figure 7A], confirming that PTGER4 signaling plays a positive role in Reh cell proliferation. Cell migration was also inhibited in a dose-dependent manner with the addition of increasing concentrations of L-161,982 [Figure 7B]. Camptothecin-induced apoptosis was unaffected by PTGER4 chemical inhibition [Figure 7C], confirming the results obtained using shRNA knockdown. These results indicate that PTGER4 transduces a positive leukemic signal enhancing cell proliferation and migration in REH cells.

\section{Discussion}

Several childhood pre-B ALL harbor the $\mathrm{t}(12 ; 21)$ translocation, yielding an ETV6-RUNX1 fusion protein thought to confer ETV6-dependant repressive activity to RUNX1 transcriptional targets[18]. Experimental evidence indicates that the ETV6-RUNX1 fusion is insufficient to initiate leukemia, which instead requires the allelic loss of the remaining wildtype ETV6 allele, suggesting that the deregulation of ETV6 transcriptional targets play an important role in leukemogenesis. However, functional targets of ETV6 involved in leukemogenesis remain elusive. Using a combination of ChIP, gene reporter assays, and loss-of-function analyses we demonstrated that SPHK1 and PTGER4 are direct transcriptional targets of ETV6 and that they have a functional role in promoting or maintaining a leukemic phenotype. Although increased expression of both these genes has been associated with many types of cancer, this is the first demonstration that their expression could be modulated by the ETV6 tumor suppressor.

\section{ETV6 binds directly to the promoter of SPHK1 and PTGER4}

ETV6 can both interact directly with the histone deacetylase co-repressor complex SMRT/N-CoR/mSin3A/HDAC and bind DNA in a sequence-specific manner [10-13]. Using ChIP analysis, we have shown that ETV6 can bind the promoter region of SPHK1 and PTGER4 loci. The molecular mechanisms used by ETV6 to

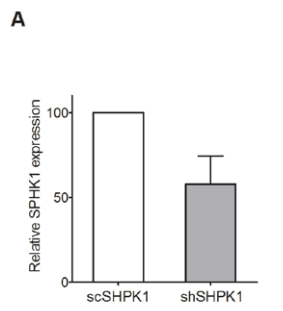

c
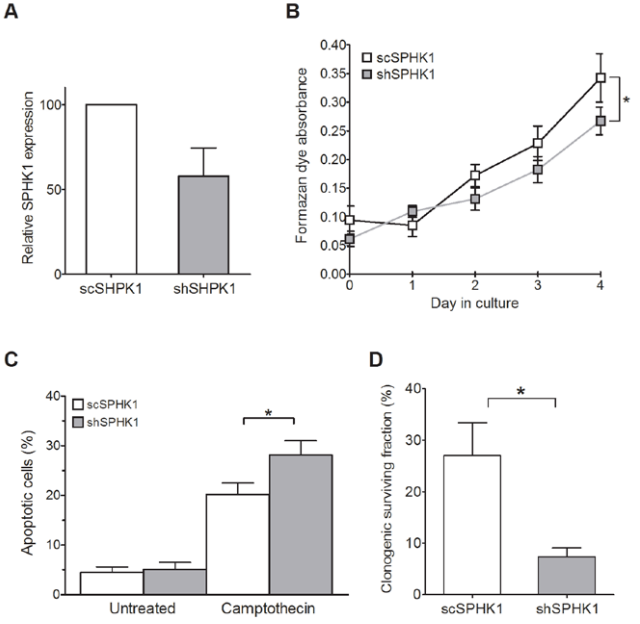

E

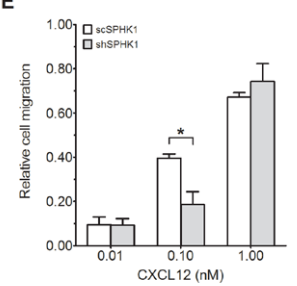

Figure 5: The ETV6-transcriptional target SPHK1 is a positive regulator of leukemia cell survival, clonogenic potential, and migration. (A) qPCR analysis of SPHK1 expression in Reh cells stably expressing a SPHK1specific shRNA (shSPHK1) or a scrambled shRNA (scSPHK1). Expression levels were normalized to $G A P D H$ and expressed relative to scSPHK1-expressing cells. Western blot analysis of SPHK1 protein levels confirms the knockdown of the endogenous protein (data not shown). (B) Knockdown of SPHK1 expression decreases Reh cell proliferation. Formazan dye absorbance is proportional to cell density (mean \pm SE). Statistical significance of differences between the linear regression slopes $\left({ }^{*}\right.$ )(days 0 to 4 ) were assessed, using scSPHK1 as reference (n = 4). (C) SPHK1 is a negative regulator of DNA damage-induced apoptosis in Reh cells. The percent of apoptotic cells was determined by adding the fractions that were Annexin $\mathrm{V}^{+} / \mathrm{PI}^{+}$and Annexin $\mathrm{V}^{+} / \mathrm{PI}^{-}$gated outside the cellular debris (mean $\pm \mathrm{SE}$ ). Statistical significance was assessed using an unpaired t-test ( $\mathrm{n}=3$ ). (D) Knockdown of SPHK1 expression decreases the clonogenic potential of Reh cells. The clonogenic surviving fraction corresponds to the plating efficiency of shSPHK1-infected cell normalized to the plating efficiency of the scSPHK1-infected Reh cells (expressed in percentage). (E) Knockdown of SPHK1 expression decreases transmigration potential of Reh cells. Relative cell migration is measured as the ratio of migrated cells/input (mean $\pm \mathrm{SE}$ ). Statistical significance was assessed using a non-parametric Mann-Whitney t-test $(n=4)$. 
regulate transcription require both its PNT protein-protein interaction domain and its ETS DNA-binding domain. In this study, we have shown that the overexpression of ETV6 in two different cell lines can actively repress the proximal promoterregion of both SPHK1 and PTGER4. This repression was dependent on both ETV6 functional domains, indicating that these genes behave like typical ETV6 transcriptional targets. Mutational analysis of the
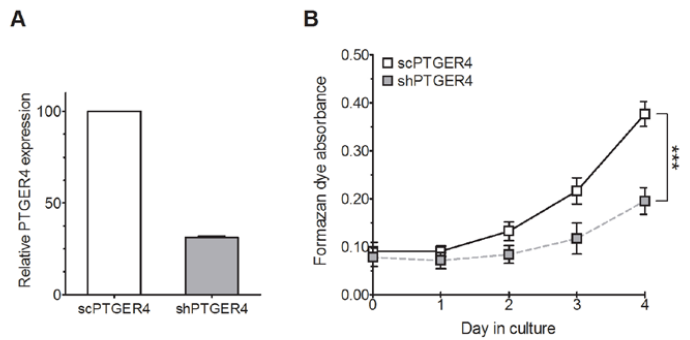

C
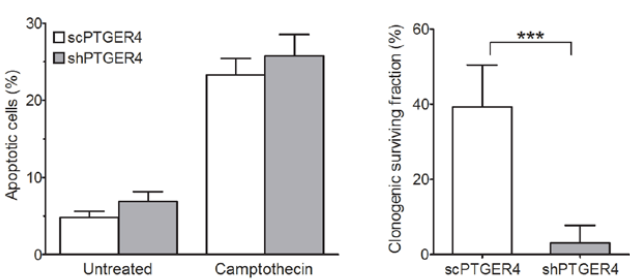

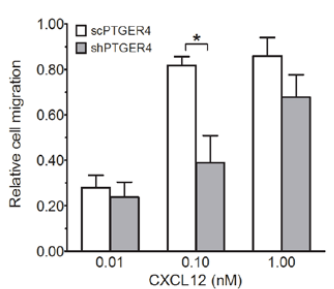

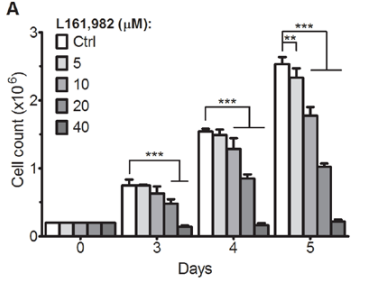

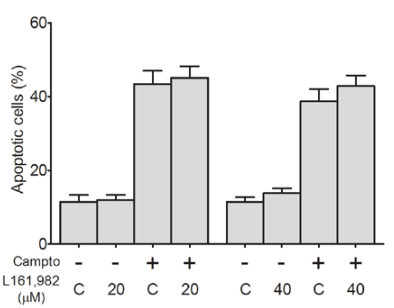

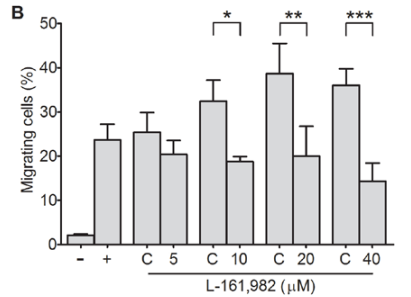

Figure 7: Inhibition of PTGER4 using a specific inhibitor reveals a functional role in the leukemic transformation of Reh cells. (A) The PTGER4 inhibitor L-161,982 prevents proliferation of Reh cells in a dose-dependent manner. The inhibitor was added at the indicated concentrations and cell counts were performed over a 5-day period. No significant difference was observed on cell proliferation exposed to the different DMSO concentration corresponding to that used with the inhibitors. Data for the DMSO control, equivalent to that of $40 \mu \mathrm{M}$ inhibitor is shown. Statistical significance was assessed using two-way ANOVA with Bonferroni's multiple comparison post-test. (B) The PTGER4 inhibitor L-161,982 inhibits the migration capability of the Reh cells through transwells containing $10 \mathrm{ng} / \mathrm{mL}$ of CXCL12 chemoattractant. The controls (C) contain DMSO concentrations equivalent to the ones used for the various inhibitor concentrations. The negative control (-) has no CXCL12 attractant, while the positive control (+) has CXCL12. (C) The PTGER4 inhibitor L-161,982 induces no cytoprotective effect to DNA damage-induced apoptosis. The percent of apoptotic cells was determined by adding the fractions that were Annexin $\mathrm{V}^{+}$and $\mathrm{PI}^{+}$gated outside the cellular debris (mean $\pm \mathrm{SE}$ ). Statistical significance for the migration and apoptosis assays was assessed using one-way ANOVA with Bonferroni's multiple comparison post-test. All experiments were repeated three time and representative results shown.

Figure 6: The ETV6 transcriptional target, PTGER4, is a positive regulator of leukemia cell survival, clonegenic potential, and migration. (A) qPCR analysis of PTGER4 expression in Reh cells stably expressing PTGER4-specific shRNA (shPTGER4) or a scrambled shRNA (scPTGER4). Expression levels were normalized to GAPDH and expressed relative to scPTGER4-expressing cells. Western blot analysis of PTGER4 protein levels confirms the knockdown of the endogenous protein (data not shown). (B) Knockdown of PTGER4 expression decreases Reh cell proliferation. Formazan dye absorbance is proportional to cell density (mean \pm SE). Statistical differences between the linear regression slopes $\left.{ }^{* * *}\right)$ were assessed (days 0 to 4$)$ using scPTGER4 as reference $(n=4)$. (C) Knockdown of PTGER4 expression has no DNA damage-induced apoptosis cytoprotective effect. The percent of apoptotic cells was determined by adding the fractions that were Annexin $\mathrm{V}^{+} / \mathrm{PI}^{+}$and Annexin $\mathrm{V}^{+} / \mathrm{PI}^{-}$gated outside the cellular debris (mean $\pm \mathrm{SE}$ ). Statistical significance was assessed using an unpaired Student's t-test $(n=3)$. (D) Knockdown of PTGER4 expression decreases the clonogenic potential of Reh cells. The clonogenic surviving fraction corresponds to the plating efficiency of shPTGER4-infected cells normalized to the plating efficiency of scPTGER4-infected Reh cells (expressed in percentage). Statistical significance was assessed using an unpaired Student's t-test (n = 3). (E) Knockdown of PTGER4 expression decreases transmigration potential of Reh cells. Relative cell migration is measured as the ratio of migrated cells/input (mean \pm SE). Statistical significance was assessed using a non-parametric Mann-Whitney t-test $(n=4)$.
EBS on the minimal promoter region still responsive to ETV6 revealed that they were required for ETV6-mediated repression. Taken together these data strongly suggest that ETV6 binds directly to the promoter of these target genes to downregulate their expression.

\section{A functional role of SPHK1 in the progression of ETV6- deficient pre-B ALL}

Since the reporter assays revealed that the promoters of both target genes could be repressed by ETV6, our functional studies were conducted in the ETV6-deficient pre-B ALL Reh cell line. We found that the downregulation of SPHK1expression reduced cellular proliferation and migration andenhancedapoptosis in response to DNA damage. SPHK1 is a lipid kinase that catalyzes the phosphorylation of sphingosine to S1P, which in turn stimulates cell proliferation, survival and migration by binding to various S1P receptors (S1PR) [38]. S1PRs activate G proteins which interact with numerous intracellular pathways including MAPK/ERKs and PI3K, involved in cell proliferation 
and survival, as well as with PLC and Rac, both involved in cell migration. Sphingosine can also be transformed into ceramide, a pro-apoptotic signal, by ceramide synthase. Increased expression of SPHK1 would therefore shift sphingosine towards S1P rather than ceramide and would consequently favor cellular proliferation, survival, and migration. Our results are consistent with the known biological function of SPHK1 and attribute a functional role to SPHK1 in the progression of ETV6-deficient pre-B ALL. We have also observed that downregulation of SPHK1 results in decreased clonogenic potential of Reh cells, suggesting SPHK1 levels regulate autonomous cell growth, which would also contribute to leukemogenesis.

Leukemia can originate from a blockage of differentiation process in early progenitors and a premature mobilization of these defective progenitors in the circulation (egress). Gradients of S1P are directly involved in hematopoietic stem cell (HSC)/ progenitor cell (HPC) egress. High concentration of S1P in the peripheral blood and low levels of S1P in the bone marrow and other tissues promote the mobilization of HPC into the circulation $[41,42]$. Recently, crosstalk has been uncovered between CXCR4/ CXCL12 pathway, responsible for the homing of the HPC to the bone marrow, and the S1P signaling pathway [43]. Increased levels of S1P activate there lease of the CXCL12 ligand from the bone marrow stroma tothe circulation and work in concert with S1P gradients to promote HPC egress from the bone marrow. Therefore, it is conceivable that increase of SPHK1 expression in ETV6-deficient ALL progenitors would favor their release from the bone marrow which, combined with the enhanced selfrenewal potential and the increase in proliferation, migration, and survival signals, would contribute to leukemogenesis.

Our findings are consistent with previous observations documenting the up-regulation of SPHK1 in many cancers, including acute leukemia [38]. Moreover, although the expression of SPHK1 has been shown to be regulated by a range of growth factors and protein kinases, this study provides the first evidence of SPHK1 regulation by a transcriptional repressor involved in childhood leukemia.

\section{A functional role of PTGER4 in the progression of ETV6-deficient pre-B ALL}

PTGER4 expression is elevated in numerous cancers including breast, colon, and endometrial adenocarcinoma and is known to enhance cell survival and cell proliferation by activating PKB(Akt), ERK1/2, and early growth response factor-1 (EGR-1) [44-47]. PTGER4 is one of four PGE2 receptors, which in turn is synthesized by COX enzymes [47]. Overexpression of COX2 has a main role in the tumorigenesis of breast and colorectal cancer [48, 49], and has been the target in several therapeutic approaches using nonsteroidal anti-inflammatory drugs (NSAIDs) similar to aspirin [50]. Because of side effects related to the use of NSAIDS, mostly affecting the heart, PTGER4 inhibitors have gained increasing attention. In this study, we have demonstrated that ETV6 directly binds to the PTGER4 promoter and represses its expression. Down-regulation of PTGER4 activity by shRNA or receptor antagonists in an ETV6-deficient cell line resulted in decreased proliferation, migration, and clonogenic potential, indicating that the increased expression of PTGER4 could contribute to leukemogenesis. These results indicate that the PGE2/PTGER4 pathway is involved in leukemogenic transformation and that increased PTGER4 receptor levels are sufficient to sensitize cells to transforming processes.

A recent study has uncovered a pivotal role for PGE2/PTGER4 signaling in HSC egress from the bone marrow. While the inhibition of PGE2/PTGER4 signaling in the stromal microenvironment causes HSC egress, inhibition of the PGE2/PTGER4 signaling within the hematopoietic cells favors myeloid hematopoietic progenitor cell, but not HSC, egress from the bone marrow [51]. It is difficult to consolidate these results with enhanced PGE2/ PTGER4 signaling in leukemic cells since it would favor homing of progenitor cells to the marrow. However, the role of PGE2/ PTGER4 in pre-B lymphoid cells was not assessed in that study and repression of this pathway in the bone marrow stroma in pre-B ALL has never been investigated. It is noteworthy that murine ETV6 hematopoietic cells are defective in homing to the bone marrow of transplanted mice [8], suggesting that ETV6deficient tumor precursor cells could egress rather than enter the bone marrow. The role of PTGER4 in the egress of these ETV6deficient tumor precursor cells remains to be examined.

Overall, this study provides evidence that deregulated expression of ETV6 transcriptional targets following the loss of ETV6 might be involved in the development of childhood pre-B ALL. Further evidence assessing the impact of PTGER4 and SPHK1 dysregulation in patients that lack functional ETV6 could provide further insights into the mechanisms underlying ETV6-mediated leukemogenesis. Furthermore, inhibition of the PGE2/PTGER4 pathway or S1P production could provide interesting avenues for the development of alternative therapeutic approaches.

\section{Funding}

This work was supported by the Canadian Institutes of Health Research (\#MOP-93518 to D.S.); and the Cancer Research Society (\#14162 to D.S.).

\section{Acknowledgements}

The authors have no potential conflicts of interest and no competing financial interests. The authors wish to thank the Canadian Institutes of Health Research and the Cancer Research Society for their financial support. At the time of the study, C.M. held a Ph.D. scholarship from the Fonds de Recherche en Santé du Québec (FRSQ). D.S. holds the François-Karl Viau Chair in Pediatric Oncogenomics and is a scholar of the FRSQ. C.M. designed and performed the reporter gene assays and the functional studies, analyzed the results and wrote the paper. K.L. and C.D. designed and performed functional studies. S.L. performed the chromatin immunoprecipitation experiments. J.L. and E.A.K. participated to the design of the experiments and revised the manuscript. C.S. and S.D. participated in the design of the experiments with inhibitors and writing the manuscript. D.S. is the principal investigator of the research and participated in the design and interpretation of the results as well as in the writing of the paper. 


\section{Conflict of interest statement}

The authors report no conflict of interest.

\section{References}

1. Linabery AM, Ross JA. Trends in childhood cancer incidence in the U.S. (1992-2004). Cancer. 2008;112(2):416-432. doi:10.1002/cncr.23169.

2.Pui CH, Carroll WL, Meshinchi S, Arceci RJ. Biology, risk stratification, and therapy of pediatric acute leukemias: an update. J Clin Oncol. 2011;29(5):551-565. doi: 10.1200/JC0.2010.30.7405.

3. Golub TR, Barker GF, Bohlander SK, Hiebert SW, Ward DC, BrayWard P, et al. Fusion of the TEL gene on 12p13 to the AML1 gene on 21q22 in acute lymphoblastic leukemia. Proc Natl Acad Sci U S A. 1995;92(11):4917-4921.

4. Shurtleff SA, Buijs A, Behm FG, Rubnitz JE, Raimondi SC, Hancock ML, et al. TEL/AML1 fusion resulting from a cryptic $t(12 ; 21)$ is the most common genetic lesion in pediatric ALL and defines a subgroup of patients with an excellent prognosis. Leukemia. 1995;9(12):19851989.

5. Poirel H, Oury C, Carron C, Duprez E, Laabi Y, Tsapis A, et al. The TEL gene products: nuclear phosphoproteins with DNA binding properties. Oncogene. 1997;14(3):349-357. doi:10.1038/sj.onc.1200829.

6. Lopez RG, Carron C, OuryC, Gardellin P, Bernard O, Ghysdael J. TEL is a sequence-specific transcriptional repressor. J Biol Chem. 1999;274(42):30132-30138.

7. Wang LC, Kuo F, FujiwaraY, GillilandDG, Golub TR, Orkin SH. Yolk sac angiogenic defect and intra-embryonic apoptosis in mice lacking the Ets-related factor TEL. EMBO J. 1997;16(14):4374-4383. doi:10.1093/ emboj/16.14.4374

8.Wang LC, Swat W, Fujiwara Y, Davidson L, Visvader J, Kuo F, et al. The TEL/ETV6 gene is required specifically for hematopoiesis in the bone marrow. Genes Dev. 1998;12(15):2392-2402.

9. Hock H, Meade E, Medeiros S, Schindler JW, Valk PJ, FujiwaraY. Tel/ Etv6 is an essential and selective regulator of adult hematopoietic stem cell survival. Genes Dev. 2004; 18(19): 2336-2341. doi:10.1101/ gad.1239604.

10. Bohlander SK. ETV6: a versatile player in leukemogenesis Seminars in cancer biology. 2005;15(3): 162-174. DOI:10.1016/j. semcancer.2005.01.008.

11. Chakrabarti SR, Nucifora G. The leukemia-associated gene TEL encodes a transcription repressor which associates with SMRT and mSin3A. Biochem Biophys Res Commun. 1999;264(3):871-877. doi:10.1006/bbrc.1999.1605.

12. Guidez F, Petrie K, Ford AM, Lu H, Bennett CA, MacGregor A, et al. Recruitment of the nuclear receptor corepressor N-CoR by the TEL moiety of the childhood leukemia-associated TEL-AML1 oncoprotein. Blood.2000;96(7): 2557-2561.

13.Wang L, Hiebert SW. TEL contacts multiple co-repressors and specifically associates with histone deacetylase-3. Oncogene. 2000;20(28):3716-3725. doi:10.1038/sj.onc.1204479.

14. Mori H, Colman SM, Xiao Z, Ford AM, Healy LE, Donaldson C, et al. Chromosome translocations and covert leukemic clones are generated during normal fetal development. Proc Natl Acad Sci U S A. 2002;99(12): 8242-8247.doi:10.1073/pnas.112218799.

15. Tsuzuki S, Seto M, Greaves M, Enver T. Modeling first-hit functions of the $\mathrm{t}(12 ; 21)$ TEL-AML1 translocation in mice. Proc Natl Acad Sci U S A. 2004;101(22): 8443-8448. doi:10.1073/pnas.040206310.
16. van der Weyden L, Giotopoulos G, Rust AG, Matheson LS, van Delft FW, Kong J, et al. Modeling the evolution of ETV6-RUNX1-induced B-cell precursor acute lymphoblastic leukemia in mice. Blood. 2011;118(4):1041-1051. doi: 10.1182/blood-2011-02-338848.

17. Sabaawy HE, Azuma M, Embree LJ, Tsai HJ, Starost MF, Hickstein DD. TEL-AML1 transgenic zebrafish model of precursor B cell acute lymphoblastic leukemia. Proc Natl Acad Sci U S A. 2006;103(41):1516615171. doi:10.1073/pnas.0603349103.

18. Hong D, Gupta R, Ancliff P, Atzberger A, Brown J, Soneji S, et al. Initiating and cancer-propagating cells in TEL-AML1-associated childhood leukemia. Science. 2008;319(5861):336-339. doi:10.1126/ science.1150648.

19. Montpetit A, Boily G, Sinnett D. A detailed transcriptional map of the chromosome 12p12 tumour suppressor locus. Eur J Hum Genet. 2002;10(1):62-71. doi:10.1038/sj.ejhg.5200766.

20. Lilljebjorn H, Soneson C, Andersson A, Heldrup J, Behrendtz M, Kawamata, N, et al. The correlation pattern of acquired copy number changes in 164 ETV6/RUNX1-positive childhood acute lymphoblastic leukemias. Hum Mol Genet. 2010;19(16):3150-3158. doi:10.1093/ $\mathrm{hmg} / \mathrm{ddq} 224$.

21. Montpetit A, Larose J, Boily G, Langlois S, Trudel N, Sinnett D. Mutational and expression analysis of the chromosome 12p candidate tumor suppressor genes in pre-B acute lymphoblastic leukemia. Leukemia. 2004;18(9):1499-1504. doi:10.1038/sj.leu.2403441.

22. Patel N, Goff LK, Clark T, Ford AM, Foot N, Lillington D,et al. Expression profile of wild-type ETV6 in childhood acute leukaemia. Br J Haematol. 2003;122(1):94-98.

23.Fenrick R, Wang L , Nip J, Amann JM, Rooney RJ, Walker-Daniels J, et al. TEL, a putative tumor suppressor, modulates cell growth and cell morphology of ras-transformed cells while repressing the transcription of stromelysin-1. Mol Cell Biol. 2000;20(16):5828-5839.

24. Rompaey LV, Potter M, Adams C, Grosveld G. Tel induces a G1 arrest and suppresses Ras-induced transformation. Oncogene. 2000;19(46):5244-5250. doi:10.1038/sj.onc.1203899.

25. Irvin BJ, Wood LD, Wang L, Fenrick R, Sansam CG, Packham G, et al. TEL, a putative tumor suppressor, induces apoptosis and represses transcription of Bcl-XL. J Biol Chem. 2003;278(47):46378-46386. doi:10.1074/jbc.M305189200

26. Anderson K, Lutz C, van Delft FW, Bateman CM, Guo Y, Colman SM, et al. Genetic variegation of clonal architecture and propagating cells in leukaemia. Nature. 2011;469(7330):356-361. doi:10.1038/ nature 09650 .

27. Fears S, Gavin M, Zhang DE, Hetherington C, Ben-David Y, Rowley JD, et al. Functional characterization of ETV6 and ETV6/CBFA2 in the regulation of the MCSFR proximal promoter. Proc Natl Acad Sci U S A. 1997;94(5):1949-1954.

28. Kwiatkowski BA, Zielinska-Kwiatkowska AG, Bauer TR Jr, Hickstein DD. The ETS family member Tel antagonizes the Fli-1 phenotype in hematopoietic cells. Blood Cells Mol Dis. 2000;26(1):84-90. doi:10.1006/bcmd.2000.0282.

29. Boily G, LaroseJ, Langlois S, Sinnett D. Identification of transcripts modulated by ETV6 expression. Br J Haematol. 2007;136(1):48-62. doi:10.1111/j.1365-2141.2006.06377.x.

30. Kwiatkowski BA, Bastian LS, Bauer TR Jr, Tsai S, ZielinskaKwiatkowska AG, Hickstein DD. The ets family member Tel binds to the Fli-1 oncoprotein and inhibits its transcriptional activity. J Biol Chem. 1998;273(28):17525-17530. 
31. Landry JR, Kinston S, Knezevic K, Donaldson IJ, Green AR, Gottgens B. Fli1, Elf1, and Ets1 regulate the proximal promoter of the LMO2 gene in endothelial cells. Blood. 2005;106(8):2680-2687. doi:10.1182/ blood-2004-12-4755.

32. Mavrothalassitis G, Ghysdael J. Proteins of the ETS family with transcriptional repressor activity. Oncogene. 2000;19(55):65246532. doi:10.1038/sj.onc. 1204045

33. Matys V, Kel-Margoulis OV, Fricke E, Liebich I, Land S, BarreDirrie A, Reuter I, et al. TRANSFAC and its module TRANSCompel: transcriptional gene regulation in eukaryotes. Nucleic Acids Res. 2006;34:D108-110. doi:10.1093/nar/gkj143.

34. Starck J, Doubeikovski A, Sarrazin S, Gonnet C, Rao G, Skoultchi A, Godet J, Spi-1/PU.1 is a positive regulator of the Fli-1 gene involved in inhibition of erythroid differentiation in friend erythroleukemic cell lines. Mol Cell Biol. 1999;19(1):121-135.

35. Tsuzuki S, Karnan S, Horibe K, Matsumoto K, Kato K, Inukai T,et al. Genetic abnormalities involved in $\mathrm{t}(12 ; 21)$ TEL-AML1 acute lymphoblastic leukemia: analysis by means of array-based comparative genomic hybridization. Cancer Sci. 2007;98(5): 698-706. doi:10.1111/j.1349-7006.2007.00443.x.

36. Cuvillier O, Pirianov G, Kleuser B, Vanek PG, Coso OA, Gutkind S, et al. Suppression of ceramide-mediated programmed cell death by sphingosine-1-phosphate. Nature. 1996;381(6585):800-803. doi:10.1038/381800a0.

37. Nava VE, Lacana E, Poulton S, Liu H, Sugiura M, Kono K, et al. Functional characterization of human sphingosine kinase-1. FEBS Lett. 2000;473(1):81-84.

38. Wallington-Beddoe CT, Bradstock KF, Bendall LJ. Oncogenic properties of sphingosine kinases in haematological malignancies. Br J Haematol. 2013;161(5):623-638. doi: 10.1111/bjh.12302.

39. Corcione A, Arduino N, Ferretti E, Pistorio A, Spinelli M, Ottonello $\mathrm{L}$, et al. Chemokine receptor expression and function in childhood acute lymphoblastic leukemia of B-lineage. Leukemia research. 2006;30(4):365-372. doi:10.1016/j.leukres.2005.07.009.

40. Cherukuri DP, Chen XB, Goulet AC, Young RN, Han Y, Heimark RL, et al. The EP4 receptor antagonist, L-161,982, blocks prostaglandin E2-induced signal transduction and cell proliferation in HCA-7 colon cancer cells. Experimental cell research. 2007;313(14):2969-2979. doi:10.1016/j.yexcr.2007.06.004.

41. Seitz G, Boehmler AM, Kanz L, Mohle R. The role of sphingosine 1-phosphate receptors in the trafficking of hematopoietic progenitor cells. Ann N Y Acad Sci. 2005;1044: 84-89. doi:10.1196/ annals.1349.01.
42. Ratajczak MZ, Lee H, Wysoczynski M, Wan W, Marlicz W, Laughlin MJ, et al. Novel insight into stem cell mobilization-plasma sphingosine1-phosphate is a major chemoattractant that directs the egress of hematopoietic stem progenitor cells from the bone marrow and its level in peripheral blood increases during mobilization due to activation of complement cascade/membrane attack complex. Leukemia. 2010;24(5):976-985. doi:10.1038/leu.2010.53.

43. Golan K, Kollet O, Lapidot T. Dynamic Cross Talk between S1P and CXCL12 Regulates Hematopoietic Stem Cells Migration, Development and Bone Remodeling. Pharmaceuticals (Basel). 2013;6(9):11451169. doi 10.3390/ph6091145.

44. Robertson FM, Simeone AM, Mazumdar A, Shah AH, McMurray JS, Ghosh S, et al. Molecular and pharmacological blockade of the EP4 receptor selectively inhibits both proliferation and invasion of human inflammatory breast cancer cells. J Exp Ther Oncol. 2008;7(4):299312.

45. Doherty GA, Byrne SM, Molloy ES, MalhotraV, Austin SC, Kay EW, et al. Proneoplastic effects of PGE2 mediated by EP4 receptor in colorectal cancer. BMC Cancer. 2009; 9:207. doi: 10.1186/1471-2407-9-207.

46. Catalano RD, Wilson MR, Boddy SC, McKinlay AT, Sales KJ, Jabbour HN. Hypoxia and prostaglandin E receptor 4 signalling pathways synergise to promote endometrial adenocarcinoma cell proliferation and tumour growth. PLoS One. 2011;6(5):19209. doi:10.1371/ journal.pone.0019209.

47. Regan JW. EP2 and EP4 prostanoid receptor signaling. Life sciences. 2003;74(2-3):143-153.

48. Greenhough A, Smartt HJ, Moore AE, Roberts HR, Williams AC, Paraskeva C, et al. The COX-2/PGE2 pathway: key roles in the hallmarks of cancer and adaptation to the tumour microenvironment. Carcinogenesis. 2009;30(3):377-386. doi:10.1093/carcin/bgp014.

49. Eberhart CE, Coffey RJ, Radhika A, Giardiello FM, Ferrenbach S, DuBois RN. Up-regulation of cyclooxygenase 2 gene expression in human colorectal adenomas and adenocarcinomas. Gastroenterology. 1984;107(4):1183-1188.

50. Brown JR, DuBois RN. COX-2: a molecular target for colorectal cancer prevention. J Clin Oncol. 2005;23(12):2840-2855. doi:10.1200/ JC0.2005.09.051.

51. Hoggatt J, Mohammad KS, Singh P, Hoggatt AF, Chitteti BR, Speth JM, et al. Differential stem- and progenitor-cell trafficking by prostaglandin E2. Nature. 2013;495(7441):365-369. doi:10.1038/nature11929.

52. Lin TL, Wang QH, Brown P, Peacock C, Merchant AA, Brennan S, et al. Self-renewal of acute lymphocytic leukemia cells is limited by the Hedgehog pathway inhibitors cyclopamine and IPI-926. PloS one. 2010;5(12):15262. doi: 10.1371/journal.pone.0015262. 